\title{
Short communication: Streptococcus canis is able to establish a persistent udder infection in a dairy herd
}

\author{
Jarosław Król, ${ }^{*}{ }^{1}$ Jan Twardoń, ${ }^{*} \ddagger$ Jacek Mrowiec, ${ }^{*} \ddagger$ Magdalena Podkowik, ${ }^{\star} \S$ Grzegorz Dejneka, ${ }^{*} \ddagger$ \\ Bogdan Dębski,\# Tadeusz Nowicki,Il and Wojciech Zalewski* \\ *Wroclaw University of Environmental and Life Sciences, Faculty of Veterinary Medicine, 50-375 Wrocław, Poland \\ †Department of Pathology, St. Norwida 31, 50-375 Wrocław, Poland \\ ‡Department of Reproduction and Clinic of Farm Animals, Pl. Grunwaldzki 49, 50-366 Wrocław, Poland \\ $\S$ Department of Food Hygiene and Consumer Health Protection, St. Norwida 31, 50-375 Wrocław, Poland \\ \#Warsaw University of Life Sciences, St. Nowoursynowska 166, 02-787 Warsaw, Poland \\ IIMilitary University of Technology, St. Gen. Kaliskiego 2, 00-908 Warsaw, Poland
}

\begin{abstract}
Bovine mastitis caused by Streptococcus canis is relatively rare. Consequently, many epidemiologic aspects of the infection, including factors that mediate crossing of host species barriers by the pathogen, infectiousness of the microorganism to the mammary gland, and the course of the disease within a herd, are still not elucidated. Therefore, the aim of the present study was to describe results of a 15-mo observation of subclinical Strep. canis mastitis on a dairy farm housing 76 lactating Holstein-Friesian cows. Upon 3 visits to the farm during a period between April 2013 and June 2014, Strep. canis was cultured from milk samples of 17 (22.4\% of the herd), $7(9.6 \%)$, and $8(11.3 \%)$ cows, respectively. The isolates obtained were characterized phenotypically by means of the API Strep identification kit (bioMérieux, Marcy l'Etoile, France), as well as genetically by using random amplified polymorphic DNA and macrorestriction analysis of the chromosomal DNA by pulsed-field gel electrophoresis. All strains displayed the same biochemical features, and the molecular methods revealed that the isolates belonged to a single clone or were very closely related. Results of the study indicate that Strep. canis is capable of causing intramammary infections of long duration, behaving in a contagious manner. Because a persistently infected cow may serve as the source of Strep. canis infection for other animals, effective control of this type of udder infection within a herd may require similar measures to those adopted in Streptococcus agalactiae eradication programs.
\end{abstract}

Received February 11, 2015.

Accepted June 8, 2015.

${ }^{1}$ Corresponding author: jaroslaw.krol@up.wroc.pl
Key words: cattle, mastitis, Streptococcus canis, pulsed-field gel electrophoresis, random amplified polymorphic DNA

\section{Short Communication}

Bovine mastitis caused by Streptococcus canis is relatively rare (Hassan et al., 2005). To date, there have been a few reports from around the world describing cases of udder infections due to Strep. canis, or less specifically, streptococci of Lancefield group G (Barnum and Fuller, 1953; Watts et al., 1984; Chaffer et al., 2005; Hassan et al., 2005; Tikofsky and Zadoks, 2005). Although carnivores (cats and dogs) are usually considered sources of these infections, some evidence suggests that subsequent cow-to-cow transmission of Strep. canis can also take place, resulting in a massive outbreak of mastitis (Tikofsky and Zadoks, 2005). Although Strep. canis has been considered an emerging bovine pathogen with possible zoonotic implications (Whatmore et al., 2001), our knowledge on the epidemiology and pathogenesis of Strep. canis mastitis remains incomplete. In particular, such issues as factors contributing to the crossing of host species barriers and the further spread of disease among cows, modes of the organism's transmission, and the course of the disease within a herd have yet to be elucidated. In the present paper, we describe results of a long-term observation on Strep. canis mastitis in a dairy herd in Poland. Our data collected over a 15-mo period may provide more insight into epidemiologic aspects of the infection.

The study was performed between April 2013 and July 2014 on a dairy farm housing 76 (with minor fluctuations) lactating Holstein-Friesian cows in Lower Silesia, Poland. For a long time, the herd had experienced multiple cases of subclinical mastitis. Animals were fed a TMR diet and housed in an open, freestall barn with a concrete floor covered with straw and rub- 
ber mats. The straw was replaced twice a week and the whole barn was cleaned thoroughly and limed once a year. Dry cows and pregnant heifers in the late stage of gestation were kept in a separate part of the barn with the same bedding as for lactating animals. The dry period ranged from 6 to 8 wk. Animals were transferred to the calving pen 3 wk before parturition. The farm participated in the national milk quality control program, including a monthly check of individual animals for SCC and concentrations of fat, protein, lactose, and ketone bodies in milk, as well as of the whole herd for bulk milk SCC. Lactating cows were milked twice daily in a parallel parlor system with 8 milking units. The average milk yield per lactating cow was $7,800 \mathrm{~kg}$ over an average 300-d lactation period. Before milking, teats were cleaned and disinfected with a lactic acid solution. A set of cloths was used for individual treatment of 40 animals; the cloths were then washed, disinfected, and used for the remaining half of the herd. Postmilking teat disinfection was performed with an iodine-based solution. Rubber gloves were routinely worn by the personnel during the milking process. Milking machines were washed and disinfected twice daily with an alkaline or acidic preparation, used alternately, and the milking machine unit liners were replaced twice a year. Visits to the farm revealed that one dog and a few cats had access to the barn.

Clinical examination of cows and bacteriologic analysis of milk samples took place for the first time in April 2013. Evaluation of udder health was performed on every lactating cow in the herd. It consisted of inspection and palpation of the udder and teats, macroscopic examination of milk, and the California Mastitis Test (CMT). Milk samples from all quarters that tested positive on the CMT were collected aseptically for bacteriologic examination. In addition, swabs from the oral cavity of a dog and 2 cats that resided on the farm, as well as from teat cups of the milking machine, were collected.

The next 2 visits to the farm took place in September 2013 and June 2014. During these visits, all the diagnostic procedures (clinical examination, CMT, and bacteriologic culture) were repeated on the whole herd. The aim of the final visit (July 2014) was to examine treatment efficacy in Strep. canis-infected cows.

Samples for microbiological examination were cooled rapidly and transported immediately (within $1 \mathrm{~h}$ of collection) to the laboratory. Milk samples (25- $\mathrm{L}$ aliquots for each plate) were inoculated on tryptic-soy agar supplemented with $5 \%$ defibrinated sheep blood and on Modified Edwards medium (Oxoid Ltd., Basingstoke, UK). Plates were incubated aerobically for 24 $\mathrm{h}$ at $37^{\circ} \mathrm{C}$. Isolated bacteria were tentatively identified based on colony and cell morphology, Gram reaction, hemolytic pattern and catalase activity. Catalasepositive and catalase-negative, gram-positive cocci were further examined by means of the ID 32 Staph and API 20 Strep identification systems (bioMérieux, Marcy l'Etoile, France), respectively. All potentially pathogenic bacteria isolated from milk samples (streptococci and staphylococci) were subject to antimicrobial susceptibility tests (using the agar disk-diffusion method) with the following antimicrobial agents: amoxicillin with clavulanic acid, cloxacillin, danofloxacin, lincomycin with neomycin, penicillin, penicillin with novobiocin, and a combination of penicillin, novobiocin, neomycin, and streptomycin. However, allocation of animals for treatment was decided by the farm's management and, for economic reasons (potential costs of milk discarded during the withdrawal time), some of the infected cows were not treated until they were dried off. Lactating cow therapy (LCT) was administered by intramammary infusion of $200 \mathrm{mg}$ of amoxicillin with $50 \mathrm{mg}$ of clavulanic acid. Dry cow therapy (DCT), administered to all 4 quarters of all animals at dry-off, consisted of either a combination of nafcillin $(100 \mathrm{mg})$, penicillin (300 mg), and dihydrostreptomycin (100 mg) or a combination of $250 \mathrm{mg}$ of ampicillin and $500 \mathrm{mg}$ of cloxacillin. The farm's management was strongly advised to remove all pets from the barn.

Isolates phenotypically assigned to Strep. canis were additionally identified by molecular methods [speciesspecific PCR with primers camp-canis-I and camp-canis II (Hassan et al., 2005) and partial sequence analysis of the $16 \mathrm{~S}$ rRNA gene]. For epidemiologic studies, a representative group of strains isolated at various times was characterized genetically using random amplified polymorphic DNA (RAPD) analysis, as well as DNA fingerprinting by pulsed-field gel electrophoresis (PFGE). For RAPD, a single oligonucleotide primer 1204, as described by Versalovic et al. (1991) for enterobacterial repetitive intergenic consensus (ERIC)-PCR, was used. The PCR amplification comprised 38 cycles of denaturation $\left(94^{\circ} \mathrm{C}\right.$ for $\left.30 \mathrm{~s}\right)$, annealing $\left(40^{\circ} \mathrm{C}\right.$ for 30 $\mathrm{s})$, and elongation $\left(72^{\circ} \mathrm{C}\right.$ for $\left.2 \mathrm{~min}\right)$. For macrorestriction analysis, bacteria grown in tryptic-soy broth were suspended in saline to obtain a density (absorbance at $600 \mathrm{~nm}$ ) of approximately 1.1. Equal aliquots of these suspensions and molten $2 \%$ low-melting-temperature agarose were mixed together and cast in plug molds. Once solidified, plugs were incubated for $4 \mathrm{~h}$ in a lysis buffer [6 m M Tris-HCl (pH 7.4), $1 \mathrm{M} \mathrm{NaCl}, 0.5 \%$ sodium lauroyl sarcosine, $0.5 \%$ Brij, $0.2 \%$ sodium deoxycholate, and $0.05 \%$ lysozyme $(\mathrm{wt} / \mathrm{vol})]$ at $37^{\circ} \mathrm{C}$, and then for $16 \mathrm{~h}$ in ESP buffer $[10 \mathrm{~m} M$ Tris- $\mathrm{HCl}(\mathrm{pH} 7.4), 1 \mathrm{~m} M$ EDTA, $1 \%$ SDS, and $0.005 \%$ proteinase $\mathrm{K}(\mathrm{wt} / \mathrm{vol})]$ at $50^{\circ} \mathrm{C}$. Gel plugs $(6 \times 2 \times 2 \mathrm{~mm})$ were digested in a buffer containing $20 \mu \mathrm{L}$ of Buffer Tango (Thermo Fisher 
Scientific Inc., Waltham, MA), $4 \mu \mathrm{L}$ of $S m a \mathrm{I}$ restriction enzyme, and $176 \mu \mathrm{L}$ of water for $4 \mathrm{~h}$ at $30^{\circ} \mathrm{C}$. Pulsedfield electrophoresis was performed using a CHEF-DR III system (Bio-Rad Polska, Warsaw, Poland) in $0.5 \times$ Tris-borate-EDTA buffer for $20 \mathrm{~h}$, at $6 \mathrm{~V} / \mathrm{cm}$ and $14^{\circ} \mathrm{C}$, with the pulse time ranging from 3 to $20 \mathrm{~s}$. Products of electrophoresis were stained with ethidium bromide and visualized using a gel documentation system.

Upon the first examination, 34 out of 76 cows (44.7\%) yielded significant numbers of pathogens responsible for mastitis (Strep. canis, Strep. uberis, Staphylococcus aureus, or CNS; Table 1). Streptococcus canis was isolated from 22 quarters in 17 cows ( $22.4 \%$ of the herd). In 13 quarters, it represented the only microorganism found; in the remaining 9 quarters, mixed infections with staphylococci were detected (Table 2). The cows infected with Strep. canis exhibited a clearly positive reaction to CMT, with SCC ranging from 158,000 to 2,995,000 cells/mL in animals infected with Strep. canis only (with an average of $1,400,000$ cells $/ \mathrm{mL}$ ) and from 748,000 to $2,395,000$ cells/mL $(1,280,000$ cells/ $\mathrm{mL}$ on average) in cows with mixed infections. Counts of Strep. canis in examined milk samples ranged from 200 to $5,000 \mathrm{cfu} / \mathrm{mL}$. All Strep. canis isolates produced small ( 0.5 to $1 \mathrm{~mm}$ in diameter) $\beta$-hemolytic colonies on blood agar and showed a synergistic Christie, Atkins, Munch-Petersen (CAMP) reaction with Staph. aureus. In contrast, they grew very poorly on Modified Edwards medium. All the isolates displayed the same biochemical properties on the API 20 Strep strip (with digit code 4073415). They produced alkaline phosphatase, arginine dihydrolase, and leucine arylamidase, as well as acid from ribose, lactose, trehalose, and starch. Weak reactions were observed for esculin hydrolysis and $\beta$-galactosidase. All strains were susceptible to all antimicrobials tested except danofloxacin, to which an intermediate susceptibility was noted. One isolate of Strep. canis was also obtained from the oral cavity of a dog living on the farm. However, it differed biochemically from those recovered from milk samples in negative reactions for esculin, arginine dihydrolase, and trehalose. All the Strep. canis strains gave a positive reaction in the PCR assay with the camp-canis primers. Species identification of the mastitic strains was also confirmed by sequence analysis of the $16 \mathrm{~S}$ rRNA gene (carried out for 3 randomly selected isolates). Swabs from teat cups tested negative for streptococci. The second survey revealed that 7 cows (9 quarters) remained culture-positive for Strep. canis (Table 3). Of them, 2 had received DCT shortly after the first visit and 5 had not been treated at all. Of the remaining animals that had been infected with the microorganism upon the first examination, 6 proved to be negative for Strep. canis (5 received DCT and 1 received no treatment in the intervening time), 2 cows were culled between April and September, and the other 2 were dried off. No new cases of Strep . canis mastitis had been detected at that time. At the third survey, Strep. canis continued to be present within the herd (Table 3). The microorganism was isolated from 9 quarters of 8 cows, but only 2 animals belonging to this group had tested positive upon the first examination 14 mo earlier. One of these 2 cows was treated twice during this period (in June and November 2013, as DCT and LCT, respectively). The second cow received therapy only once, as DCT (in October 2013). The remaining 6 cows diagnosed with Strep. canis at the third survey had neither been infected with this bacterium nor tested positive to CMT during surveys carried out previously. Interestingly, one of the newly infected animals had calved (as a first-calf heifer) as recently as $5 \mathrm{~d}$ before milk sample collection. Of the cows that had cultured positive at the third visit, 5 were then administered with LCT, 1 was culled, and 2, close to the dry period, were left untreated. Resampling of the 5 animals after completion of the antibiotic therapy (July 2014) yielded no microorganisms (Table 3).

The RAPD analysis of the Strep. canis isolates recovered upon the first survey revealed that most (20 out of 22) belonged to one clone and the 2 remaining isolates (418C and 347D) displayed a strong genetic relationships to that clone, differing by only one additional fragment at $270 \mathrm{bp}$. The same RAPD patterns were obtained after completion of the 14-mo observa-

Table 1. Descriptive bacteriological and clinical results from milk samples collected from a Polish dairy herd during 3 herd visits

\begin{tabular}{lccc}
\hline Item & $\begin{array}{c}\text { First survey } \\
\text { (April 2013) }\end{array}$ & $\begin{array}{c}\text { Second survey } \\
\text { (September 2013) }\end{array}$ & $\begin{array}{c}\text { Third survey } \\
\text { (June 2014) }\end{array}$ \\
\hline Number of lactating cows in the herd & 76 & 73 & 71 \\
Number (\%) of cows with clinical mastitis & $13(17.1)$ & $7(9.6)$ & $5(7.0)$ \\
Number (\%) of cows with subclinical mastitis ${ }^{2}$ & $29(38.2)$ & $27(37.0)$ & $23(32.4)$ \\
Bulk milk SCC $\left(\times 10^{3}\right.$ ) & 1,003 & $721(34.2)$ & 702 \\
Number (\%) of culture-positive cows & $34(44.7)$ & $25(32.4)$ \\
${ }^{1}$ Visible changes in milk in at least one quarter. & & & \\
${ }^{2}$ Elevated SCC; no changes in milk. & & &
\end{tabular}


Table 2. Bacterial isolates (no. of quarters) from milk samples collected from a Polish dairy herd during 3 herd visits

\begin{tabular}{lccc}
\hline Bacteria isolated & First survey \\
(April 2013) & $\begin{array}{c}\text { Second survey } \\
\text { (September 2013) }\end{array}$ & $\begin{array}{c}\text { Third survey } \\
\text { (June 2014) }\end{array}$ \\
\hline Streptococcus canis & 13 & 4 & 9 \\
Staphylococcus aureus & 13 & 9 & 8 \\
Streptococcus uberis & 6 & 3 & 3 \\
Streptococcus canis + Staphylococcus epidermidis & 4 & 2 & 0 \\
Streptococcus canis + Staphylococcus sp. & 4 & 0 & 0 \\
Staphylococcus epidermidis & 2 & 2 & 1 \\
Staphylococcus chromogenes & 2 & 1 & 1 \\
Staphylococcus xylosus & 2 & 0 & 0 \\
Streptococcus dysgalactiae & 1 & 0 & 0 \\
Streptococcus canis + Staphylococcus haemolyticus & 1 & 0 & 0 \\
Staphylococcus sciuri & 1 & 0 & 0 \\
Staphylococcus haemolyticus & 1 & 3 & 0 \\
Streptococcus canis + Staphylococcus sciuri & 0 & 1 & 0 \\
Staphylococcus sp. & 0 & 1 & 5 \\
Streptococcus canis + Staphylococcus aureus & 0 & 2 & 0 \\
Trueperella pyogenes & 0 & 1 & 1 \\
\hline
\end{tabular}

${ }^{1}$ Staphylococcus sp. = isolates identified as Staphylococcus microti by $16 \mathrm{~S}$ rRNA gene sequencing.

tion period, while analyzing representative isolates recovered at the different examination dates (Figure 1). All 6 Strep. canis strains, isolated for the first time in 2014, belonged to the predominant genotype. The close genetic relationship of the bovine Strep. canis isolates recovered over the whole period, confirming a clonal character of the outbreak, was also shown by means of PFGE (Figure 2). Both genetic methods revealed that the isolate recovered from the dog's mouth differed distinctly from those causing mastitis.

Implementation of mastitis control programs in many countries has eliminated or greatly reduced infections of the bovine udder caused by "classical" pathogens such as Streptococcus agalactiae (Andersen et al., 2003). However, numerous other microorganisms, including other streptococci and CNS, have increasingly been involved in the infections so that mastitis is still a serious economic problem. Outbreaks of mastitis caused by Strep. canis have been described by several authors (Chaffer et al., 2005; Hassan et al., 2005; Tikofsky and Zadoks, 2005). In addition, there were infrequent reports on udder infections caused by group G streptococci before Strep. canis was established as a separate species (Barnum and Fuller, 1953; Watts et al., 1984). In general, such infections have been relatively rare and little information on the epidemiology of Strep. canis mastitis is available. In the present study, we describe a case of multiple udder infections caused by this microorganism on a dairy farm. Although other microorganisms responsible for mastitis (Strep. uberis, Staph. aureus, CNS) were isolated at various frequencies and sometimes in mixed culture, the most prevalent pathogen was Strep. canis. We found that the microorganism can cause long-term, chronic mastitis and that a few infected cows may constitute a continuous source of infection for the whole herd. Streptococcus canis can be successfully identified by both biochemical (API 20 Strep) and genotype-based tests; however, some properties (positive CAMP reaction and the ability, to some degree, to hydrolyze esculin) could lead to misidentification when classical (phenotypic) diagnostic methods are used. Our Strep. canis strains grew poorly on Edwards medium and this fact could also affect the routine diagnosis of mastitis.

Streptococcus canis mastitis in cows is often thought to result from transmission of the organism from cats or dogs. For example, Tikofsky and Zadoks (2005) detected the same bacterial strain in milk samples and in a cat with sinusitis. Direct transmission was also very likely to occur in our case, considering the free access of pets to the barn. However, because the only nonmastitic Strep. canis strain isolated by us (originating from a dog) belonged to an apparently different genotype, the real source of the infection in cows remains unknown. Interestingly, Strep. canis mastitis may also appear without any evidence of cats or dogs in the barn (Chaffer et al., 2005), indicating that the microorganism could also be introduced indirectly into the herd, possibly on the skin of farm personnel.

Results of our study indicate that Strep. canis is capable of spreading from cow to cow in a contagious manner. The microorganism is commonly known as a commensal of the skin and mucosal membranes of carnivores and there are no reports on its long-term survival and multiplication in the environment. Therefore, it seems highly unlikely that fomites may be the reservoir of Strep. canis for as long as 14 mo. Because the pets - a putative origin of the infection described 
KRÓL ET AL.

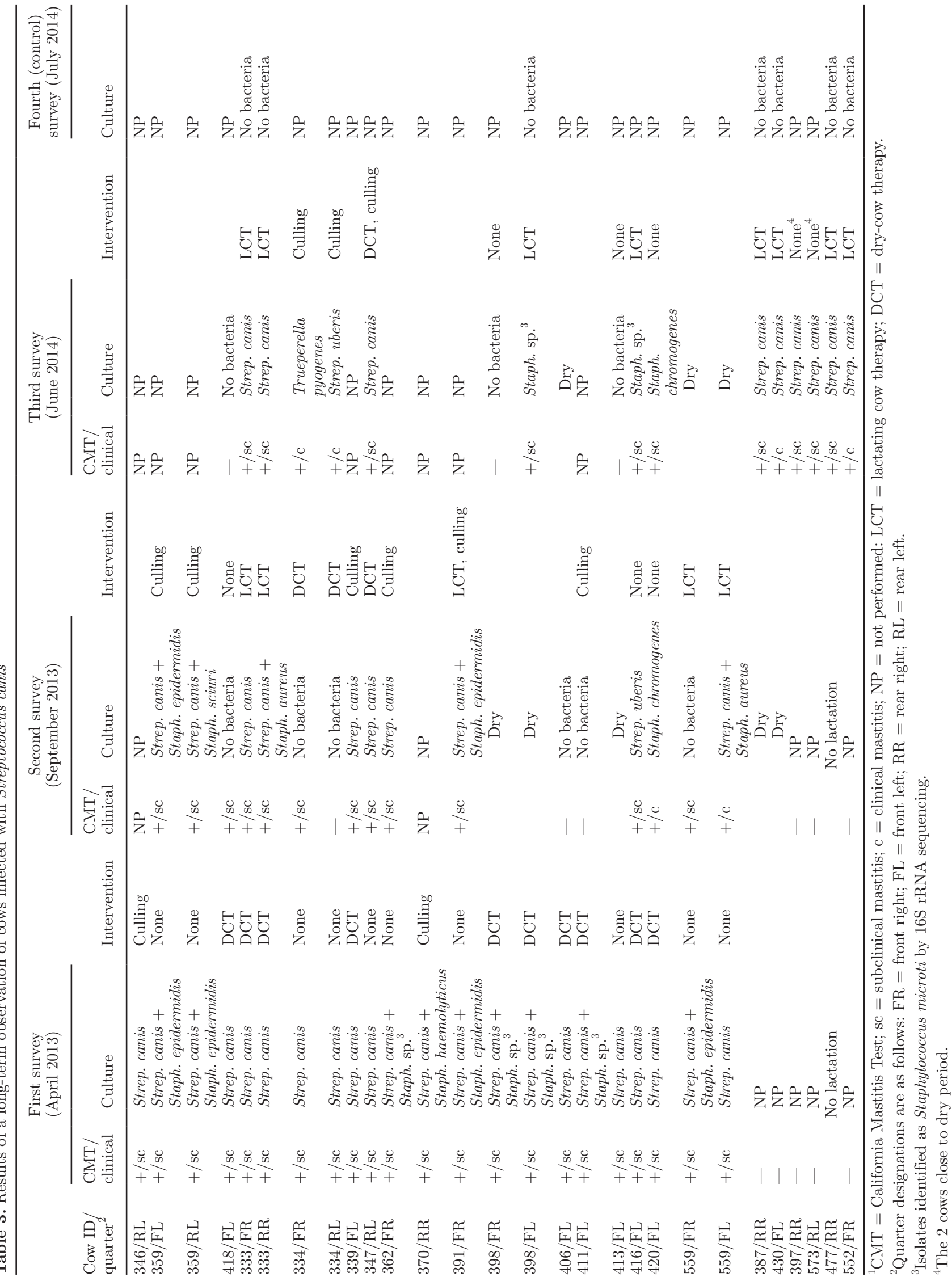




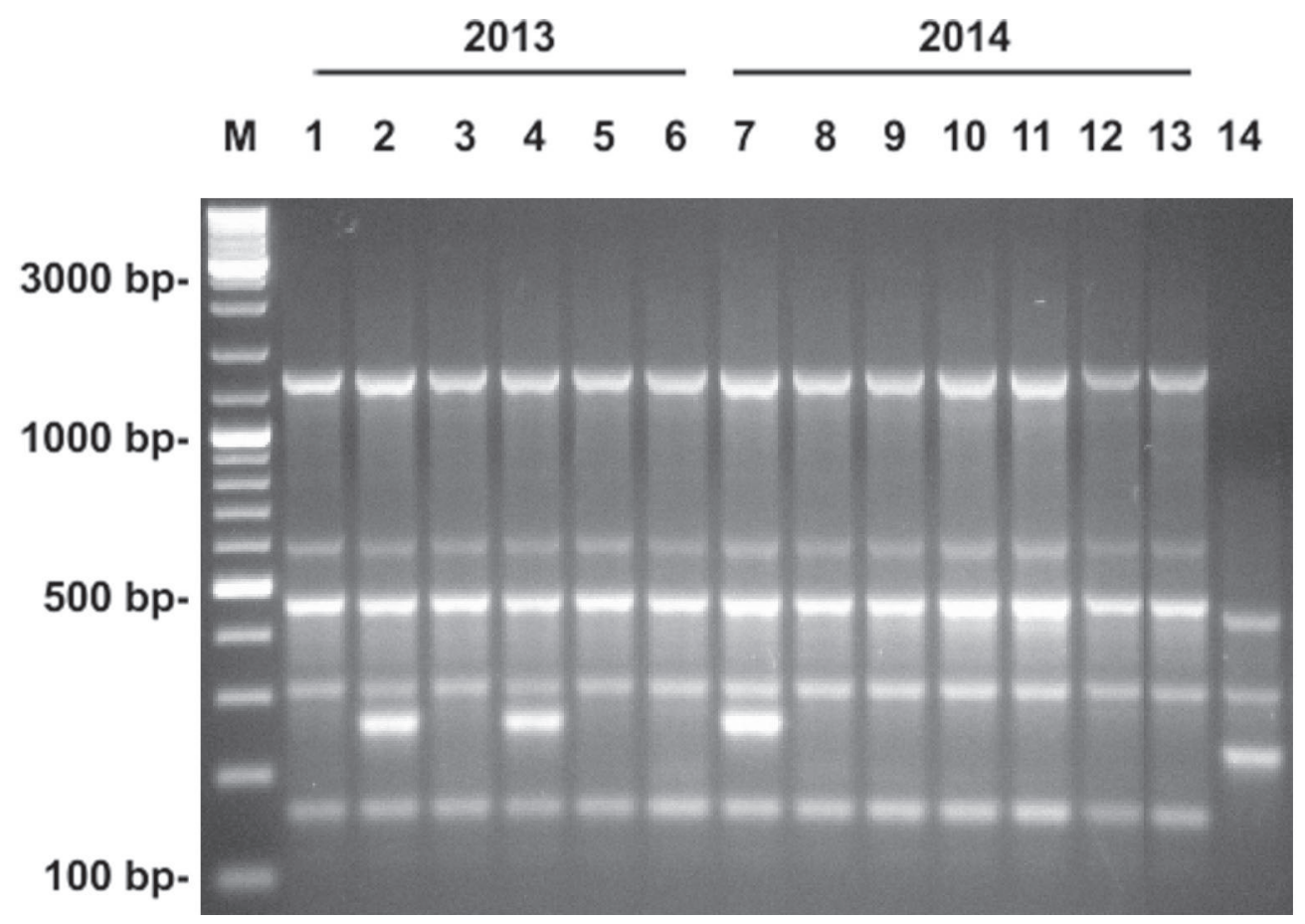

Figure 1. Random amplified polymorphic DNA (RAPD) analysis of representative Streptococcus canis isolates analyzed in the present study (designation of the bovine isolates corresponds to barn numbers and quarters of the cows infected). M = Gene Ruler DNA Ladder Mix (Thermo Fisher Scientific, Waltham, MA); lanes 1 to $6=$ strains isolated from milk samples in 2013 ( $1=406 \mathrm{FL} ; 2=418 \mathrm{FL} ; 3=416 \mathrm{FL} ; 4=347 \mathrm{RL}$ $5=333 \mathrm{FR} ; 6=387 \mathrm{RR})$; lanes 7 to $13=$ strains isolated from milk samples in $2014(7=347 \mathrm{RL} ; 8=552 \mathrm{FR} ; 9=430 \mathrm{FL} ; 10=397 \mathrm{RR} ; 11=$ 573RL; $12=477 \mathrm{RR} ; 13=333 \mathrm{FR}$ ); lane $14=$ the isolate from a dog living in the barn. Quarter designations are as follows: FR $=$ front right; $\mathrm{FL}=$ front left; $\mathrm{RR}=$ rear right; $\mathrm{RL}=$ rear left.

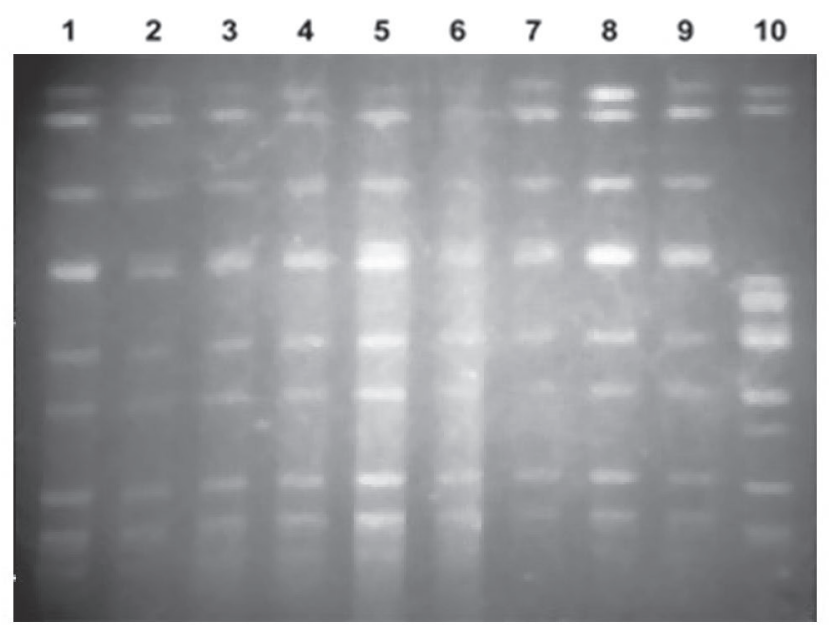

Figure 2. Pulsed-field gel electrophoresis restriction patterns of chromosomal DNA from the Streptococcus canis isolates analyzed in the present study (designation of the bovine isolates corresponds to barn numbers and quarters of the cows infected). Lanes 1 to $4=$ strains isolated from milk samples in $2013(1=359 \mathrm{FL} ; 2=362 \mathrm{FR}$; $3=559 \mathrm{FR} ; 4=333 \mathrm{FR}$ ); lanes 5 to $9=$ strains isolated from milk samples in $2014(5=397 \mathrm{RR} ; 6=573 \mathrm{RL} ; 7=347 \mathrm{RL} ; 8=333 \mathrm{FR} ; 9$ $=430 \mathrm{FL}$ ); lane $10=$ the strain isolated from a dog living in the barn Quarter designations are as follows: $\mathrm{FR}=$ front right; $\mathrm{FL}=$ front left; $\mathrm{RR}=$ rear right; $\mathrm{RL}=$ rear left. here - were removed from the barn immediately after the first recognition of Strep. canis in milk samples, the chronically infected cows remained the only possible source of this microorganism for animals infected in the period between the second and third survey. In addition, the results of genotyping of our Strep. canis isolates strongly support the notion of contagiousness. According to common belief, in the case of contagious transmission, all IMI should be attributed to a limited number of strains (Zadoks et al., 2001). In our case, the 2 Strep. canis clones (as detected by RAPD assay) differing by an additional 270-bp fragment undoubtedly originated from a common ancestor, and this genetic diversity may have been the result of a mutation.

At present, little information is available about the possible duration of Strep. canis mastitis either in individual cows or at the whole-herd level. In one of the cases described previously, the microorganism was detected in 26 cows and still present in 2 animals upon resampling after 6 mo (Chaffer et al., 2005). However, the data provided do not clearly indicate whether the 2 cows were reinfected during that interval or whether the infection was refractory to the previous treatment. In addition, lack of molecular typing of the bacterial isolates precludes a detailed epidemiologic analysis. 
More insight into this issue was provided by the work of Tikofsky and Zadoks (2005), who investigated an outbreak of Strep. canis mastitis for a similar half-year period. Those researchers found that the organism was able to persist for that time in most untreated cows and that the rate of spontaneous cure was relatively low (reported to be 9\%). Implementation of strict prevention measures as well as treatment or culling of infected animals resulted in a marked reduction but no complete elimination of the outbreak. In the case described here, we found that Strep. canis mastitis could have a much longer duration, the same genotype of the microorganism being detectable for more than a year.

Previous reports and our findings indicate that, once established within a herd, Strep. canis may be difficult to eradicate. This type of mastitis has been characterized by a marked degree of infectivity (usually multiple cases are detected on a farm and udder infection can appear very shortly after the first calving) as well as persistency, either at the herd level or in individual cows. Although the microorganism has been found susceptible to many antimicrobial agents tested in vitro (Chaffer et al., 2005; Hassan et al., 2005; Tikofsky and Zadoks, 2005), the treatment of S. canis-infected cows has occasionally failed. For example, Chaffer et al. (2005) estimated the cure rate for infected quarters to be $89 \%$, but lower figures $(87.5 \%$ for dry cow therapy and $65 \%$ for lactating cow therapy) were detected by Tikofsky and Zadoks (2005). In our case, one of the cows infected failed to respond to 2 series of treatments, thus being the possible source of infection for other animals in the period between the second and third surveys. This observation suggests that detection of long-term carriers and re-examination of cows treated for Strep. canis mastitis may be crucial in regard to successful control of the infection.

In conclusion, our findings reveal that Strep. canis is likely to adapt quite easily to the bovine udder, being able to establish long-lasting herd infections. This microorganism displays many other epidemiologic attributes that are essentially the same as those recognized for Strep. agalactiae (Keefe, 1997), including high infectivity, high within-herd prevalence, and relatively low self-cure rate. Because Strep. canis mastitis may pose therapeutic problems at the herd level, effective control and prevention of the condition may require the same measures as those adopted in eradication programs for Strep. agalactiae and other major mastitis pathogens (Barkema et al., 2009). In particular, detection and therapy of all animals infected, identification of cows that are nonresponsive to the initial treatment, complete dry-cow therapy, and proper postmilking teat dip all seem to be essential parts of a successful management program for Strep. canis-infected herds.

\section{ACKNOWLEDGMENTS}

The research was supported by the Ministry of Science and Higher Education, Warsaw, Poland (Grant No. NN308576640). Publication supported by Wroclaw Centre of Biotechnology (Wroclaw, Poland), program "The Leading National Research Centre (KNOW) for years 2014-2018."

\section{REFERENCES}

Andersen, H. J., L. H. Pedersen, F. M. Aerestrup, and M. Chriél. 2003. Evaluation of the surveillance program of Streptococcus agalactiae in Danish dairy herds. J. Dairy Sci. 86:1233-1239.

Barkema, H. W., M. J. Green, A. J. Bradley, and R. N. Zadoks 2009. The role of contagious disease in udder health. J. Dairy Sci. 92:4717-4729.

Barnum, D. A., and D. S. Fuller. 1953. Report on an outbreak of chronic mastitis in cattle caused by a Streptococcus of Lancefield's group G. Can. J. Comp. Med. 17:465-472.

Chaffer, M., S. Friedman, A. Saran, and A. Younis. 2005. An outbreak of Streptococcus canis mastitis in a dairy herd in Israel. N. Z. Vet. J. 53:261-264.

Hassan, A. A., Ö. Akineden, and E. Usleber. 2005. Identification of Streptococcus canis isolated from milk of dairy cows with subclinical mastitis. J. Clin. Microbiol. 43:1234-1238.

Keefe, G. P. 1997. Streptococcus agalactiae mastitis: A review. Can. Vet. J. 38:429-437.

Tikofsky, L. L., and R. N. Zadoks. 2005. Cross-infection between cats and cows: Origin and control of Streptococcus canis mastitis in a dairy herd. J. Dairy Sci. 88:2707-2713.

Versalovic, J., T. Koeuth, and R. Lupski. 1991. Distribution of repetitive DNA sequences in eubacteria and application to fingerprinting of bacterial genomes. Nucleic Acids Res. 19:6823-6831.

Watts, J. L., S. C. Nickerson, and J. W. Pankey. 1984. A case study of Streptococcus group G infection in a dairy herd. Vet. Microbiol. 9:571-579.

Whatmore, A. M., K. H. Engler, G. Gudmundsdottir, and A. Efstratiou. 2001. Identification of isolates of Streptococcus canis infecting humans. J. Clin. Microbiol. 39:4196-4199.

Zadoks, R. N., H. G. Allore, H. W. Barkema, O. C. Sampimon, Y. T. Gröhn, and Y. H. Schukken. 2001. Analysis of an outbreak of Streptococcus uberis mastitis. J. Dairy Sci. 84:590-599. 